\title{
Real-time monitoring of cell response to drug stimulation by 2D-SPR Sensor: An approach to study neuronal differentiation
}

\author{
Tanveer Ahamd Mir ${ }^{1}$ and Hiroaki Shinohara, ${ }^{1,2}$ \\ ${ }^{1}$ Course of Life Information and System Science, Graduate School of Innovative Life Science for \\ Education, University of Toyama, 3190 Gofuku, Toyama 930-8555, Japan. \\ ${ }^{2}$ Field of Life, Information System Sciences, Graduate School of Science and Engineering for \\ Research, University of Toyama, 3190 Gofuku, Toyama 930-8555, Japan. \\ Correspondence should be addressed to T. A. Mir, (mirtanveer0699@gmail.com) \\ and H. Shinohara (hshinoha@eng.u-toyama.ac.jp)
}

\begin{abstract}
There is a growing demand for the development of new bio-analytical technique that is capable of monitoring neuronal differentiation non-invasively, in real-time and without any fluorescent or radioactive probes. To fulfill these promises, efficient and easy protocols to monitor neuronal differentiation have to be developed. In this study, we demonstrated a rapid and label-free detection method to monitor neuronal differentiation by high-resolution 2D-surface plasmon resonance sensing technology with a CCD camera to analyzing refractive index change of PC12 cells [1]. We have successfully monitored the intracellular translocation of PKC in PC12 cells by 2D-SPR and found that the cells treated with differentiation factor, nerve growth factor (NGF) showed a remarkable enhancement of SPR response to muscarine and carbacol stimulation. Our results suggested that 2DSPR imager might be applicable to monitor neuronal differentiation and to study the expression state of the specific receptors in living state.
\end{abstract}

Key words: 2D-SPR, PC12 cell, neuronal differentiation, cell response, PKC translocation.

\section{Introduction}

Over the last decades, the concept of differentiation assessment, whereby immature cells may be stimulated to develop into their mature phenotype, aroused considerable interest. Differentiation of PC12 cells is most often assessed by semiquantitative or quantitative morphological methods. These methods can include determination of cell size, the number of cells exhibiting processes (neurites), and the extent of neurite growth or neurite length [2]. Other existing methods for dynamic probing of neuronal differentiation are currently available e.g. immunoblotting analysis, autoradiography, and PCR based differential display [3-5]. All these methods of detecting biomolecules have been implemented to assay for cell extracts, and they are not suitable for real-time study of neurons or could be limited by the need for complex microscopic configurations.

Compared to these no imaging techniques, a widely used imaging technique is based on fluorescence detection, which can image individual cells labeled with dye molecules [6-7].
Therefore, real-time and label-free techniques are highly desired because they remove possible effects of the labels on the functions of neurons. More importantly, unlike the labelbased methods that measure the labels, labelfree techniques directly measure the intrinsic physical and biological characteristics of the cells, providing additional information. Our laboratory is interested in investigating the realtime monitoring of intracellular alterations accompanying morphological changes during neuronal development and degeneration in model cells at individual cell level. As such, our goal is to combine the powerful biosensing techniques as differentiation signaling elements [8].

Here, we explore the utility of twodimensional surface plasmon resonance (2DSPR) biosensor for the detection of neuronal differentiation in living state [9]. On this basis, the PC12 cell line is chosen as a representative example of a model cells, which is one of the most widely used neuronal precursor cell lines for research on examining the mechanisms underlying neuronal differentiation [10]. These 
cells respond to nerve growth factor (NGF), undergoing morphological change that generates phenotype similar to that of sympathetic neurons characterized by neurite outgrowth, suggesting that this system may facilitate the evaluation of the changes in biochemical composition that accompany the NGF-induced differentiation of these cells [1112]. We tested both NGF expressed and endogenous drug response to signaling pathway of PKC translocation in PC12 cells via reflection intensity change upon SPR measurements. The reflection intensity of cell regions were continuously monitored using a real-time 2D-SPR sensing system with CCD camera. The individual cells were imaged as they scatter surface plasmon waves. The capability of detecting single neuronal cells allows us to monitor cell-surface interactions and to determine the refractive index of individual living cells and their changes upon stimuli.

Our result suggests that differentiated cells are indeed more sensitive in response to extracellular stimuli. Since NGF-treated cells induce much defined SPR signals, it is possible to harness this information as a functional and biologically relevant readout for neuronal differentiation study. Finally, major aspect of using non-invasive and real-time sensing to monitor the neuronal differentiation allow for the interfacing of SPR sensing without cell damage. Such methods are highly desirable for the use of neuronal cells in biosensors or in neuronSPR technology.

\section{Material and method}

PC12 cells were cultured on the bare gold surface of the SPR sensor chip with DMEM including $10 \%$ horse serum, $5 \%$ fetal bovine serum and $1 \%$ penicillin-streptomycin, and were incubated at $37^{\circ} \mathrm{C}$ in an atmosphere of $5 \%$ carbon dioxide. After 1 day of subculture, the cells were treated with $80 \mathrm{ng} / \mathrm{ml}$ nerve growth factor (NGF) for differentiation for 2-5 days. NGF treatment was performed by DMEM with only $1 \% \mathrm{HS}$.

\section{D-SPRI Experimental Setup}

The 2D-SPR experiments were performed using a (2D-SPR 04A, NTT-AT.Co., Ltd., Japan) surface plasmon resonance apparatus possessing a collimator and a CCD camera with four magnification lens: $1 \times, 2 x, 4 \times$ and $7 x$ (Fig.1). The detailed experimental procedures have been described in our previous report [1]. Briefly, the 2D-SPR interrogation system consists of a diode laser $(630 \mathrm{~nm})$ system for generating a parallel light beam that illuminates a 2D-SPR sensor chip and a CCD camerabased receive system for recording changes in the intensity of the light beam reflected from the sensor chip. For SPR-neuronal signal analysis, the gold sensor surface was modified with polyL-lysine and incubated for over night in a humid environment, and thereafter the same sensor chips were utilized for making cell culture samples. We observed reflection intensity changes at different developmental stages of PC12 cells cultured on the surface of a sensor chip under SPR imaging system at suitable incident angle.

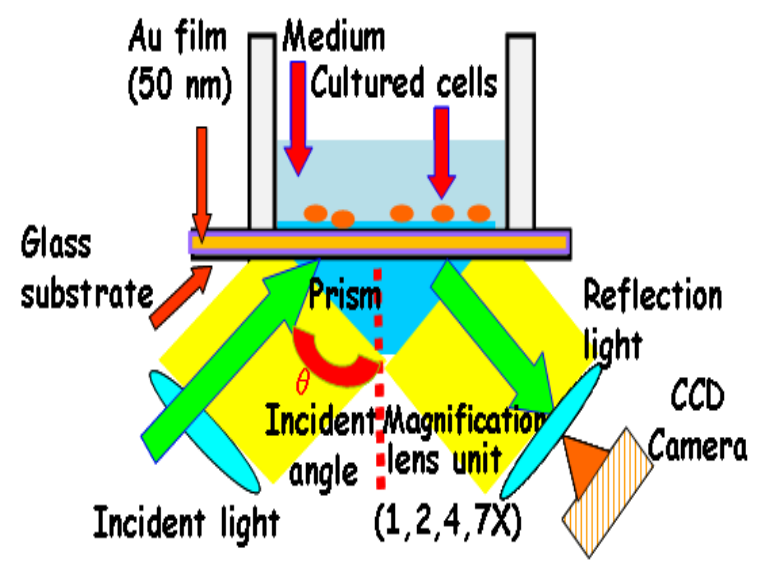

Fig.1 Observation system with 2D-SPR.

\section{Monitoring of cell proliferation and differentiation by phase-contrast microscopy}

PC12 cells were seeded onto a gold sensor chip coated with poly-L-lysine at an initial density of $4.0 \times 10^{5}$ cells per sample for experiment before differentiation, and $3.0 \times 10^{5}$ cells per sample for experiments after differentiation, respectively. The proliferation of NGF-treated and untreated PC12 cells was estimated daily over a period of up to 5 days and the morphological changes of the cell were observed by phase-contrast microscopy (I x 70 Olympus) equipped with CCD camera to capture the images (data not shown). Briefly, in both NGF-untreated with high serum and NGFtreated cell culture conditions, the sensor chips were incubated for 24hrs, 2days and 4days, respectively. At the end of the incubation period samples were collected and their surface plasmon resonance signal was evaluated.

\section{Results and discussion}

To study the PKC translocation triggered by drug-stimulation for cell differentiation signaling, the 2D-SPR integration system was used to monitor in real-time muscarine and carbacolinduced response in PC12 cells. The effect of 
NGF on PC12 culture was analyzed by observing reflection intensity changes at individual cell level under the consideration that after differentiation, PC12 cells stop proliferation, and different response could be observed. To compare the results obtained for both NGF-treated and NGF-untreated cells, proliferating PC12 cells (treated with $10 \% \mathrm{HS}$ ) showed less response upon muscarine

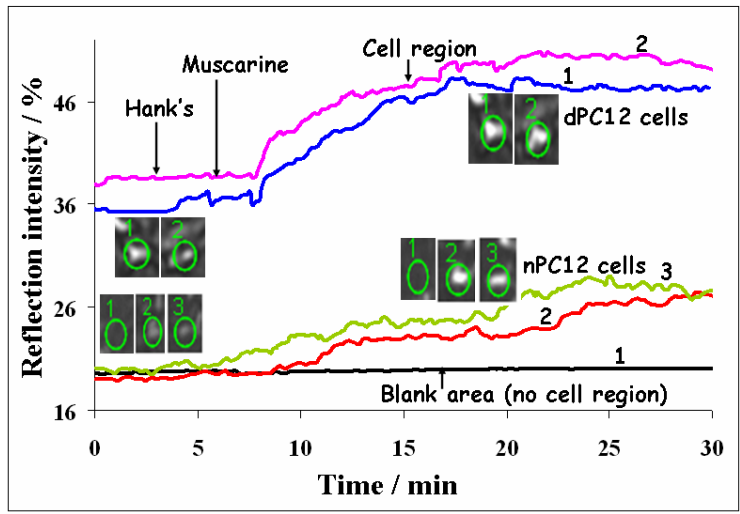

stimulation as shown in Fig 2.

Fig. 2 2D-SPR response of differentiated and nondifferentiated PC12 cells to muscarine.

The dependence of reflection intensity change induced by stimulation with various concentrations of muscarine in the NGF-treated and untreated PC12 cells is shown in Fig 3.

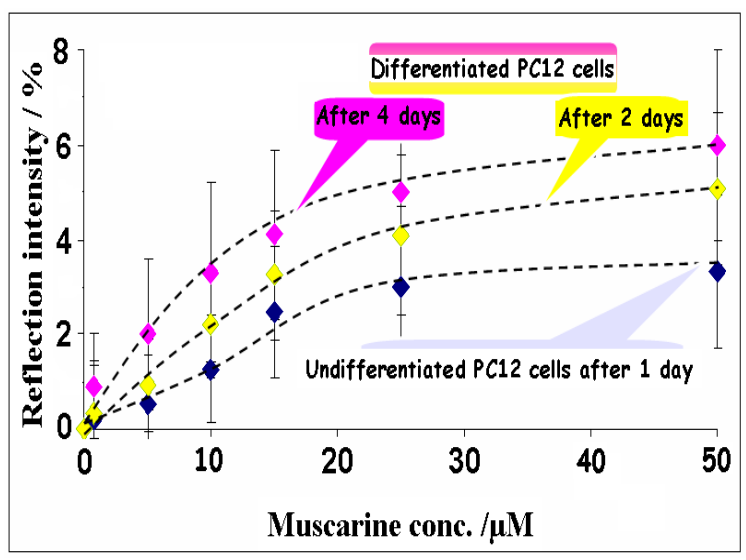

Fig. 3 Muscarine-induced SPR response for PC12 cells observed at different developmental stages.

Conventional approaches to study neuronal differentiation have shown that NGF-treated PC12 cells have much higher muscarinic acetylcholine receptors as compared with proliferating cells. Therefore, our results are consistent with previous findings and suggesting that the muscarine and carbacolinduced SPR signal enhancement might be based on increased expression of muscarinic acetylcholine receptors. Our finding suggested that a remarkable enhancement of SPR response for PKC stimulants attributed to PKC translocation might be a good signature of differentiation in PC12 cells.

\section{Conclusion}

We have demonstrated a real-time and labelfree detection of neuronal differentiation of PC12 cells with two-dimensional surface plasmon resonance imaging technology. A major pragmatic implication of this current work is the differentiation of PC12 cells by $2 \mathrm{D}-\mathrm{SPR}$ signatures, eliminating the need for biochemical interventions (e.g. antibody labeling, chemiselective stains). Such a no labeling optical approach is particularly useful for cellular differentiation at individual cell level. On the basis of the data presented here, we conclude that 2D-SPR based method present a real-time and label-free approach for separating undifferentiated PC12 cells and their differentiated derivatives based on objective biophysical criteria. This technology is expected to be critical for future applications in label-free 2D-SPR monitoring for differentiation of various precursor cells.

\section{References}

[1] M. Horii, H. Shinohara, Y. Irebe, M. Suzuki, M, Analyst 3, 837-841 (2011).

[2] Y. Obara, N. Nakahata and Y. Ohizumi, Brain Res., 1998, 806, 79.

[3] P. W. Gunning, G. E. Landerth, P. Layer, M. Ignatius and E. M. Shooter, J. Neuroscience 4, 368-379 (1981).

[4] H. Nakagawa, M. Yoshida and S. Miamoto, Journal of Biochemistry 127, 113 -119 (2000).

[5] N. Aoki, Y. Y. Aoki and A. Ullrich, Journal of Biochemistry 271(46), 29422-29426 (1996).

[6] K. C. Appell and D. S. Barefoot, Biochemical Journal 263, 11-18 (1989).

[7] E. S. Wachman, R. E. Poage, J. R. Stiles, D. L. Farkas and S. D. Meriney, J. Neuroscience 24(12), 2877-2885(2004).

[8] T. A. Mir, H. Shinohara, and Y. Shimizu Analytical Methods 3, 837-841, 2011

[9] M. Tanaka, T. Hiragun, T. Tsutsui, Y. Yanase, H. Suzuki and M. Hide, Biosensors and Bioelectronics 23, 1652-1658 (2008).

[10] L.A. Greene, A.S,Tischler, Proc Natl Acad Sci. 73, 2424-2428 (1976).

[11] G. Dauod, E. Rassart, A. Masse and J. Lafond, Journal of Physiololgy 571(3), 537-553 (2006).

[12] L.A. Greene, A.S,Tischler, Advances in Cell Neurobiology 3, 373-414 (1982). 